\title{
How we decide when to immobilize the wrist after stable osteosynthesis of displaced distal radius fracture
}

\author{
Kilian M, Heger T, Simkovic P, Jacko P, Szaboova A, Simko P \\ Traumatology Clinic, Slovak Medical University and UNB, Hospital Acad. Ladislav Derer, Bratislava, Slovakia. \\ mini083cx@gmail.com
}

\begin{abstract}
PURPOSE OF THE STUDY: Fractures of the distal radius are frequently associated with injuries of the scapholunate (SL) and lunotriquetral (LT) ligaments. Our study is aimed at revealing their hidden lesions by employing a fast and accessible fluoroscopic identification.

PATIENTS AND METHODS: We investigated 40 patients who were indicated for plate osteosynthesis of distal radius fracture. After completing the osteosynthesis, the procedure was concluded with a wrist arthrography. The patients with SL and LT interval lesions had their wrists immobilized by a plaster splint while patients with normal findings with an elastic bandage. The patients were followed up for 12 months after the surgery. The functional results were evaluated by Mayo wrist score.

RESULTS: The intra-operative examination identified $62.5 \%$ of patients with lesions of SL and/or LT interval, and $37.5 \%$ of patients were lesion-free. The Mayo wrist scores after 3, 6 and 12 months in patients whose wrists were not immobilized were $72,86.3$, and 86.3 , respectively. The latter scores in the group of patients with external immobilization were $54.4,82$, and 84.8 , respectively. The difference between the groups was significant three months after the surgery. After six and twelve months, the difference became negligible. CONCLUSION: The exclusion of hidden lesions allows earlier rehabilitation, while in patients with signs of lesions, it is appropriate to immobilize the wrist (Tab. 1, Fig. 3, Ref. 31). Text in PDF www.elis.sk KEY WORDS: distal radius fractures, scapholunate ligament, lunotriquetral ligament, fluoroscopic, diagnosis.
\end{abstract}

\section{Introduction}

The fractures of distal radius are often associated with injuries of scapholunate and lunotriquetral ligaments while the triangular fibrocartilaginous complex (TFCC) injuries are frequently present as well. The distal radial fractures occur together with injuries of the scapholunate (SL) ligament in 13.6-68 \% of cases (1-6), while those with concomitant injuries of the lunotriquetral (LT) ligament occur in $8.5-15 \%$ of cases $(3,5,7)$. Static instabilities can be observed on radiographic images in form of widened intercarpal intervals and increased scapholunate angle, which is caused by concomitant injuries of intrinsic and extrinsic ligaments of the wrist (8). However, by means of posterior-anterior and lateral radiographic projections standardly used in cases of fractures of the distal radius, it is not possible to reveal pre-dynamic and dynamic instabilities occurring in association with isolated injuries of intrinsic ligaments. Fresh fractures of the distal radius do not allow other diagnostic tools to be used, such as thorough clinical examination of the wrist, dynamic radiographic examination of the wrist or standard acute examination by means of magnetic resonance (MR). As long, as these lesions remain hidden, instabili-

Traumatology Clinic, Slovak Medical University and UNB, Hospital Acad. Ladislav Derer, Bratislava, Slovakia

Address for correspondence: A. Szaboova, Traumatology Clinic, Slovak Medical University and UNB, Hospital Acad. Ladislav Derer, Bratislava, Limbová 5, SK-833 05 Bratislava, Slovakia. ties tend to occur, and in turn, gradual degenerative changes in the joint tend to develop (9). A precise diagnosis of these concomitant injuries is currently possible only by means of arthroscopy of the wrist. The fractures of the distal radius are however very frequent injuries, and it is impossible to accept a situation, in which a precise diagnosis can be achieved only at clinics with the privilege of being able to perform arthroscopy. The recognition of these hidden concomitant injuries and their subsequent treatment are a precondition for good final functional results. Our study's goal is to evaluate a procedure that can reveal these hidden wrist lesions occurring in association with fractures of the distal radius by employing a fast and generally available fluoroscopic diagnostic tool.

\section{Patients and methods}

During the period of 27 months, we investigated 40 patients who were indicated for surgery due to a fracture of the distal radius. The prospective study cohort contained 30 women and 10 men at an average age of $55 \pm 14.9$ (21-83) years. The patients were indicated for surgery due to instable extra-articular and dislocated intra-articular fractures of the distal radius. All fractures were treated by open reduction and volar-plate osteosynthesis with an angle-stable implant. In neither of patients enrolled in the study did the simple radiographic imaging reveal a widening in SL and/or LT interval. Excluded from the study were patients with open fractures, fractures with static instability, fractures of carpal bones, and fractures requiring other methods of fixation, as well 
as incompliant patients and those with associated instability of the distal radioulnar joint that was identified during the surgery.

After completing the osteosynthesis of the distal radius, the procedure was concluded with arthrographic examination of the midcarpal joint. We studied the flow of the contrast medium through SL and LT intervals. For the purpose of arthrography, we used a small 25 -gauge needle and $76 \%$ iodine contrast medium (Urgorafin, Bayer AG, Germany). In order for the entire capitate-lunate space to be filled up gradually, and contrast medium to fill up the SL and LT spaces, the space between triquetral, lunate and hamate bones was chosen as the site for midcarpal joint puncture, which was then done under radiographic control. The fluoroscopic control enabled us to watch the entry of the contrast substance into the radio-carpal space, which marked the lesions in intrinsic ligaments. We examined the wrists in prone position, namely in neutral, radial (widening of LT interval) and ulnar deviations (widening of SL interval) (Figs 1-3). The lateral projection was used only for the purpose of evaluating the depth down to which the tip of the needle went in order not to introduce the contrast medium iatrogenically into the adjacent dorsal or volar soft tissues and thus disable the subsequent arthrographic evaluation.

In line with the results of arthrography, the patients with pathological arthrographic findings had their wrists immobilized by means of a plaster splint for a period of six weeks. Patients with normal arthrographic findings had their wrists fixed postoperatively with elastic bandage only (until removal of sutures). Patients were followed up for a period of 12 months after the surgery and functional results were evaluated by the system of Mayo wrist scoring in 3-, 6- and 12-month intervals. The final evaluation of median and average values of clinical parameters was done according to normality of data distribution. Subsequently, we compared the resulting values of only two variables at a time by means of unpaired t-test. In case of $p<0.05$, the result was considered significant.

\section{Results}

Out of the total number of 40 fractures treated surgically (40 patients), we identified 25 patients $(62.5 \%)$ with the intra-operative finding of penetration of the contrast medium through SL and/or

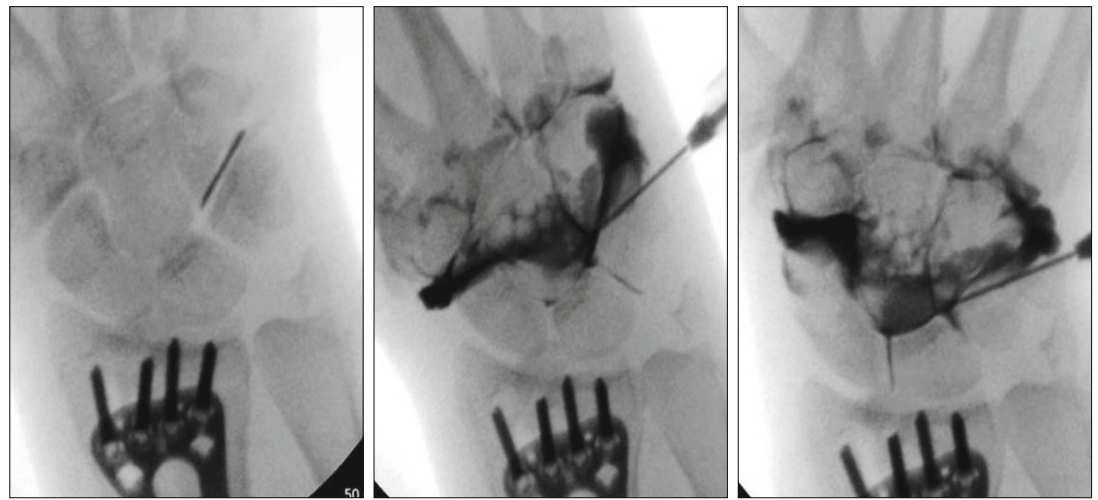

Fig. 1. Fracture of distal radius after osteosynthesis with no penetration of contrast medium through SL and LT intervals.
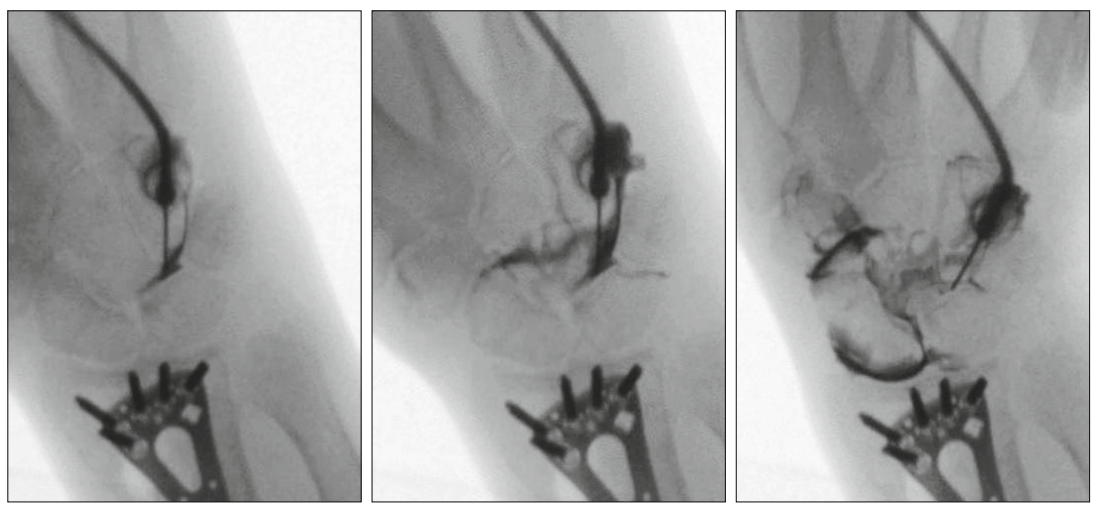

Fig. 2. Fracture of distal radius after osteosynthesis with penetration of contrast medium through SL interval.
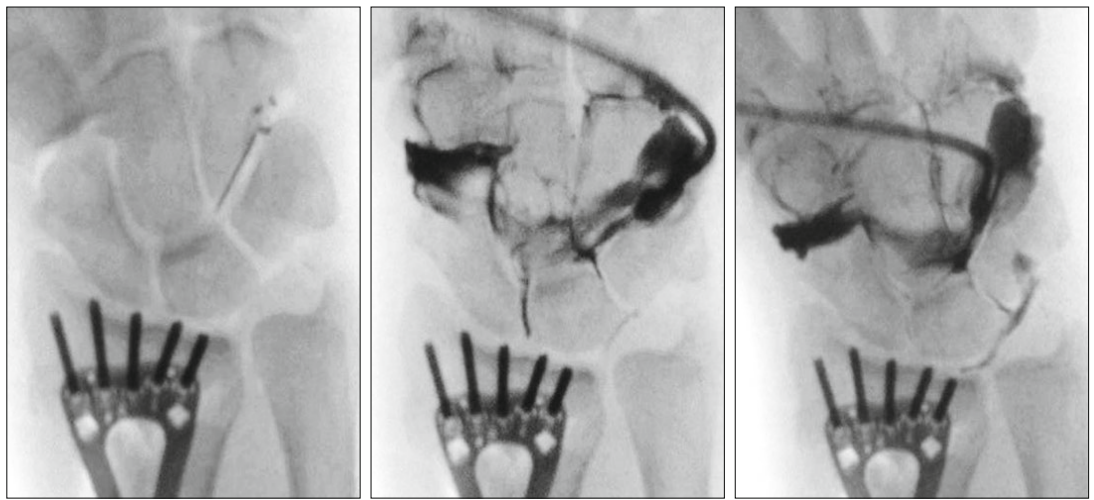

Fig. 3. Fracture of distal radius after osteosynthesis with penetration of contrast medium through LT interval.

LT intervals. In 8,8 and 9 of the latter 25 patients, the contrast medium penetrated solely through the SL interval, solely through LT interval, and through both SL and LT intervals, respectively. In 15 patients $(37.5 \%)$, there was no sign of penetration of the contrast agent (Tab. 1). The incidence of SL and/or LT lesions assessed based upon the penetration of contrast medium and type of fracture according to AO classification is shown in Table 1. The average duration of arthrography of the midcarpal joint was 3.13 $\pm 0.04(2.0-5.3)$ minutes. The Mayo wrist scores after 3, 6 and 
Tab. 1. AO classification, penetration of contrast medium from the midcarpal joint through SL and/or LT interval and Mayo wrist scores 3,6 and 12 months after surgery.

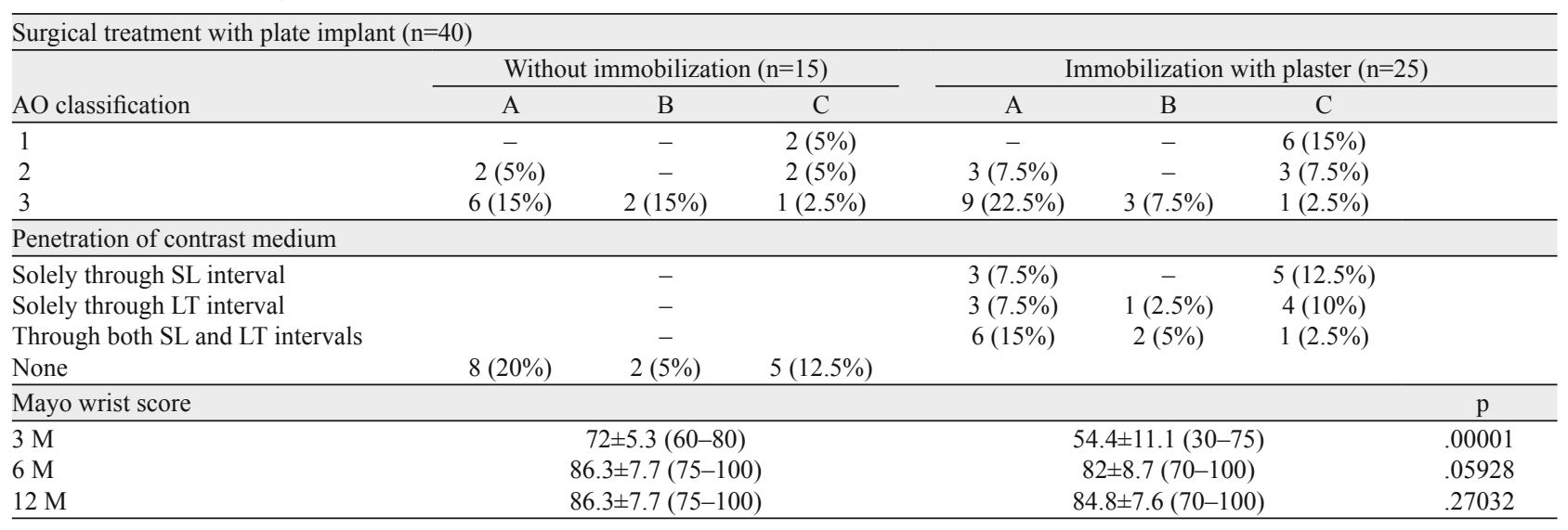

12 months are shown also in Table 1 . The difference in the scores between the groups was significant 3 months after the surgery. The latter difference became negligible 6 months after the surgery and stayed this way at the time point of 12 months after surgery (Tab. 1).

\section{Discussion}

The fractures of distal radius are frequent, and the incidence of concomitant lesions of SL and LT ligaments is evidently higher than it was originally thought to be. The operative treatment of instable dislocated extra-articular and intra-articular fractures of the distal radius by means of angle-stable plates is currently regarded as part of the standard procedure while we can expect other techniques in the future $(10,11)$. The concomitant injuries of SL and LT intervals are standardly diagnosed by a combination of clinical, radiological, and CT/MR arthrography examinations, but arthroscopy has currently become the best option. However, due to the fact, that the clinical examination of a painful wrist with fracture of distal radius is of low informative value, the radiographic examination remains the cornerstone of the diagnostic procedure. The preoperative radiographic examination often reveals only the static instabilities. In order to reveal dynamic carpal instabilities, some authors reevaluate perioperatively the radiographic examination by means of fluoroscopy (1, 12). This technique of measurement (although currently performed with advanced machines) can, however, yield particular mistakes and therefore requires mathematical recalculation or calibration of the monitored image (1). Although some carpal instabilities can be revealed with a thorough clinical examination of the wrist when carried out immediately after finishing the stable osteosynthesis, such assessment, similarly to that done by means of dynamic radiographic examination carried out after osteosynthesis, requires a relatively high level of experience with diagnostic procedures and treatment of carpal instabilities. Then there is the method of MR examination and its modifications which, under our conditions, are not indicated in a routine urgent setting. The diagnosis of carpal instabilities is important from the aspect of further management, namely in view of the indication for surgical intervention when the lesions of intercarpal ligaments are evident, or at least in terms of further additional external plaster fixation when the latter lesions are hidden. The golden standard of the diagnosis of concomitant carpal lesions is wrist arthroscopy which is also used at our clinic. However, it is not being standardly used in the setting of distal radius fractures. Neither other workplaces have the option of utilizing it, mainly due to its unavailability as well as because it is associated with a time-consuming procedure (1). However, in order to be able to treat the intercarpal lesions adequately, or at least to improve the treatment by external plaster fixation, it is appropriate to make an attempt to detect them. It is necessary mainly because there are still unanswered questions as to whether the hidden lesions of ligaments might progress, and whether, even after stable osteosynthesis of the distal radius performed with the use of angle-stable plate, it is still necessary to immobilize the wrist postoperatively. Jones et al reported that in their retrospective study conducted in a sample of 221 patients with fractures of the distal radius, the scapholunate instabilities evaluated by means of posterior-anterior and lateral projections appeared as unusual either in the setting of acute wrist fracture or in association with the progression of hidden injuries of ligaments - even despite early rehabilitation (13). This is, why some authors do not immobilize fractured wrists at all (14); others fix them for 1-2, 3, 4 weeks or longer $(13,15-17)$. Some studies have yielded better short-term results as to the function and movement range of the wrist in patients who had their wrists immobilized for 1 or 3 weeks as compared with those who had their wrists fixed for 6 weeks (18). From the long-term aspect, though, there were no differences in the results (18). Also, the study of Quadlbauer et al showed that early immobilization of the wrist after the surgery due to fractures of the distal radius by means of a volar locking plate was a reliable method that led to a better function of the wrist. In that study, they achieved a better movement range in the sagittal and frontal planes, rotation of underarm and grasping force in 6 months, 9 weeks, 6 weeks and 6 months, respectively. The functional score was also better at 6 weeks after surgery, namely in patients with early mobilization of the wrist as compared with those who were immobilized for 5 weeks. After one year, though, there were no 
significant differences between the groups and the only significant difference was in the muscular force of the limb that was surgically treated and rehabilitated early as compared with the limb that was not operated on (19). On the other hand, another study shows that the initiation of exercise 6 weeks after surgery did not lead to a decrease in the movement range of the wrist as assessed 3 and 6 months after surgery when compared to the group of patients who started exercising after 2 weeks (20). The surgical treatment of dislocated fractures of the distal radius with thermoplastic fixation for 6 weeks appears to be sufficient for achieving good results after one year of follow-up, even also with MR findings of complex lesions in the wrist which are very frequent at the time of accident, and it seems that only a small proportion of them decompensates later (21).

In our opinion, the aspect of earliness of the return of function can have social and economic impacts on patients as well as on the healthcare provider. Therefore, the attempt to exclude the hidden lesions of SL and LT ligaments spares the patients of having to undergo additional fixation of the wrist while their return to common activities can be faster. At our clinic, we were thus making informed decisions in line with the results of arthrographic examination of the midcarpal joint which was performed to detect ligament injuries of SL and LT intervals in association with fractures of the distal radius. All patients in whom we found the penetration of contrast medium through SL and/or LT interval had their wrists fixed with a plaster splinter for 6 weeks, whereas the wrists of the rest of the patients were not fixed, and after suture removal, they started with early rehabilitation. In our cohort, we identified a total of $62.5 \%$ of cases with lesions of SL and/or LT interval. This incidence is comparable with those found in other studies $(5,7$, 22). None of the lesions of SL and/or LT interval were evaluated as severe (cases with unambiguous lesions were excluded from our cohort) and as such they were not indicated for surgical treatment and treated only with a plaster splinter.

The arthrographic assessment of intercarpal lesions has been known already since 1961 (23). Because of its low sensitivity, though, it has been gradually pushed into the background. In the work of Cooney, the author studied 20 patients with chronic pain in the wrist, who underwent radio-carpal, midcarpal or TFCC arthrography, arthroscopy and arthrotomy. By means of arthrography, they identified 8 and 7 cases with lesions of the scapholunate and lunotriquetral ligaments, respectively, while arthroscopy led to proving 7 and 9 cases of lesions of the scapholunate and lunotriquetral ligaments, respectively. In total, though, the arthrographic findings correlated with arthroscopic findings in 12 of 20 cases (24). Roth and Haddad analyzed 37 patients with chronic ulnar pain of the wrist, namely by means of arthrography and arthroscopy. In 17 patients, the arthrographic findings were evaluated as normal, whereas in 8 of them, the findings were confirmed to be abnormal (25). In the study of Chung et al, out of 150 patients with chronic pain in the wrist, there were 44 who had normal arthrographic findings. Yet, the arthroscopic examination revealed abnormalities in 35 of the latter patients $(80 \%)$. The sensitivity of triple arthrography was $74 \%$ and specificity was $56 \%$ (26). On the other hand, in their retrospective study, Kim et al used the technique of dynamic cine-arthrography in 38 patients with chronic pain of the wrist, where they applied the contrast medium into the radiocarpal joint, and after passive exercise, they investigated the penetration of the contrast medium into the midcarpal joint as well as the flow through the distal radio-ulnar joint. They further compared the results of dynamic cine-arthrography with the findings achieved with MR arthrography. The levels of general sensitivity and specificity of the dynamic cine-arthrography and MR arthrography were similar relative to the diagnosis of lesions of intrinsic ligaments (SL ligaments: sensitivity $66.7 \%$ vs $80 \%$, and specificity $100 \%$ vs $95.7 \%$; LT ligament: sensitivity $75 \%$ vs $75 \%$ and specificity $94.1 \%$ vs $91.2 \%$ ) (27). In our study, we were not focused on patients with chronic wrist pain but on those with fractures of the distal radius, and we were searching for hidden lesions of SL and LT ligaments in order to make the decision as to whether to apply additional fixation of the wrist or to recommend early mobilization. We are aware of the fact, that minute tears in SL and LT ligaments are present also in asymptomatic patients with no history of wrist injury (28, 29). The incidence of asymptomatic communication between midcarpal and radiocarpal joints is $17 \%$ in the age category of 15-29 years, and it increases up to $50 \%$ in the age category of $50-70$ years (30). Hence, this does not mean that each communicating defect necessarily represents a criterion for abnormality (31). However, in our cohort, we were not able to identify asymptomatic patients. All arthrographically confirmed cases of communication between midcarpal and radiocarpal joints were considered abnormal by us. All such cases were always indicated for additional fixation of the wrist with plaster splinter. In the rest of cases, we considered abnormalities to be excluded, and the patients were indicated for early mobilization even though we are aware that arthrography does not have to necessarily reveal older lesions, through which the contrast medium does not flow. However, after a 1-year follow-up, no difference in wrist function was to be found between our two groups. This could be a sign that in our cohort, there was no case of an unambiguously false-positive arthrographic finding. Also, in our study, we did not assess the associated TFCC lesions, which are also common. The latter topic is being tackled by us in a separate study of distal radius fractures. When considering further therapy after receiving the arthrographic results in association with distal radius fractures, it is necessary to take into consideration also the typical age distribution. In older patients, the osteoporotic terrain often decreases the risk of associated injury of carpal ligaments. However, if early postoperative mobilization of the wrist is impelled in cases of fractures that took place in an osteoporotic terrain, the risk that osteosynthesis may become a failure, increases. Even though, in our study, we proceeded in line with the results of arthrography independently of age, in our opinion, in older patients, also in cases with negative arthrographic findings, it is necessary for the limb to be fixed, and thus to enable still healing of the fragile fracture.

\section{Conclusion}

Despite the fact, that sensitivity and specificity of arthrography are debatable, it is possible to take into consideration its diagnostic value in cases of distal fracture of the radius in order to detect the associated injury of carpal ligaments. The procedure takes a rela- 
tively short time, and its cost is low. It is also commonly available at clinics routinely dealing with the surgical treatment of distal fractures of the radius. In a selected group of patients, early mobilization of the wrist can lead to superior functional results that can be achieved in an earlier fashion.

\section{References}

1. Gajdoš R, Pilný J, Pokorná A. Konkomitantné poranenia skafolunátneho väzu pri zlomeninách distálneho rádia - perioperačná diagnostika a výsledky liečby [Injury to the Scapholunate Ligament in Distal Radius Fractures: Peri-Operative Diagnosis and Treatment Results]. Acta Chir Orthop Traumatol Cech 2016; 83 (5): 336-343.

2. Schädel-Höpfner M, Böhringer G, Junge A, Celik I, Gotzen L. Arthroskopisch gesicherte Begleitverletzungen des skapholunären Bandapparates bei distalen Radiusfrakturen. Handchir Mikrochir Plast Chir 2001; 33 (4): 229-233.

3. Geissler WB, Freeland AE, Savoie FH, McIntyre LW, Whipple TL. Intracarpal soft-tissue lesions associated with an intra-articular fracture of the distal end of the radius. J Bone Joint Surg Am 1996; 78 (3): 357-365.

4. Peicha G, Seibert F, Fellinger M, Grechenig W. Midterm results of arthroscopic treatment of scapholunate ligament lesions associated with intra-articular distal radius fractures. Knee Surg Sports Traumatol Arthrosc 1999; 7: 327-333.

5. Richards RS, Bennett JD, Roth JH, Milne K Jr. Arthroscopic diagnosis of intra-articular soft tissue injuries associated with distal radial fractures. J Hand Surg Am 1997; 22 (5): 772-776.

6. Mrkonjic A, Lindau T, Geijer M, Tägil M. Arthroscopically diagnosed scapholunate ligament injuries associated with distal radial fractures: a 13- to 15-year follow-up. J Hand Surg Am 2015; 40 (6): 1077-1082.

7. Lindau T, Arner M, Hagberg L. Intraarticular lesions in distal fractures of the radius in young adults. A descriptive arthroscopic study in 50 patients. J Hand Surg Br 1997; 22 (5): 638-643.

8. Čižmář I, Kovařík J, Vlček M, Korpa P. Artroskopická resekce a spongioplastika skafolunátního kloubu v léčbě chronické dynamické skafolunátní nestability. Acta Chir Orthop Traumatol Cech 2018; 85 (1): 34-39.

9. Pilný J, Kubes J, Hoza P, Mechl M, Visna P. Skafolunátní nestability zápestí po zlomeninách distálního radia. Acta Chir Orthop Traumatol Cech 2007; 74 (1): 55-58.

10. Kilian M, Simkovic P, Zamborsky R, Skoda A, Labas P. What are the ideal screw lengths in volar locking plate fixation for distal radius fractures? Bratisl Med J 2019; 120 (7): 505-509.

11. Zamborsky R, Kilian M, Jacko P, Bernadic M, Hudak R. Perspectives of 3D printing technology in orthopaedic surgery. Bratisl Med J2019; 120 (7): 498-504.

12. Kwon BC, Baek GH. Fluoroscopic diagnosis of scapholunate interosseous ligament injuries in distal radius fractures. Clin Orthop Relat Res 2008; 466 (4): 969-976.

13. Jones VM, Everding NG, Desmarais JM, Soong MC. Scapholunate instability after distal radius volar plating. Hand (N Y) 2015; 10 (4): 678-682.

14. Fowler JR, Ilyas AM. Prospective evaluation of distal radius fractures treated with variable-angle volar locking plates. J Hand Surg Am 2013; 38 (11): 2198-2203.

15. Karantana A, Downing ND, Forward DP et al. Surgical treatment of distal radial fractures with a volar locking plate versus conventional percutaneous methods: a randomized controlled trial. J Bone Joint Surg Am 2013; 95 (19): 1737-1744.

16. Arora R, Lutz M, Deml C, Krappinger D, Haug L, Gabl M. A prospective randomized trial comparing nonoperative treatment with volar locking plate fixation for displaced and unstable distal radial fractures in patients sixty-five years of age and older. J Bone Joint Surg Am 2011; 93 (23): 2146-2153.

17. Osti M, Mittler C, Zinnecker R, Westreicher C, Allhoff C, Benedetto KP. Locking versus nonlocking palmar plate fixation of distal radius fractures. Orthopedics 2012; 35 (11): 1613-1617.

18. Watson N, Haines T, Tran P, Keating JL. A Comparison of the Effect of One, Three, or Six Weeks of Immobilization on Function and Pain After Open Reduction and Internal Fixation of Distal Radial Fractures in Adults: A Randomized Controlled Trial. J Bone Joint Surg Am 2018; 100 (13): 1118-1125.

19. Quadlbauer S, Pezzei C, Jurkowitsch J et al. Early Rehabilitation of Distal Radius Fractures Stabilized by Volar Locking Plate: A Prospective Randomized Pilot Study. J Wrist Surg 2017; 6 (2): 102-112.

20. Lozano-Calderón SA, Souer S, Mudgal C, Jupiter JB, Ring D. Wrist mobilization following volar plate fixation of fractures of the distal part of the radius. J Bone Joint Surg Am 2008; 90 (6): 1297-1304.

21. Gologan R, Ginter VM, Haeffner A, Obertacke U, Schreiner U. 1 -Year outcome of concomitant intracarpal lesions in patients with dislocated distal radial fractures: a systematic assessment of 78 distal radial fractures [published correction appears in Arch Orthop Trauma Surg 2016 Mar; 136 (3): 433]. Arch Orthop Trauma Surg 2016; 136 (3): 425-432.

22. Ogawa T, Tanaka T, Yanai T, Kumagai H, Ochiai N. Analysis of soft tissue injuries associated with distal radius fractures. BMC Sports Sci Med Rehabil 2013; 5 (1): 19. Published 2013 Sep 2.

23. Kessler I, Silberman Z. An experimental study of the radiocarpal joint by arthrography. Surg Gynecol Obstet 1961; 112: 33-40.

24. Cooney WP. Evaluation of chronic wrist pain by arthrography, arthroscopy, and arthrotomy. J Hand Surg Am 1993; 18 (5): 815-822.

25. Roth JH, Haddad RG. Radiocarpal arthroscopy and arthrography in the diagnosis of ulnar wrist pain. Arthroscopy 1986; 2 (4): 234-243.

26. Chung KC, Zimmerman NB, Travis MT. Wrist arthrography versus arthroscopy: a comparative study of 150 cases. J Hand Surg Am 1996 Jul; 21 (4): 591-594.

27. Kim TY, Lee GY, Kim BH, Park JW, Seo BK, Cha SH. The Usefulness of Dynamic Cine-Arthrography for Wrist Instability as Correlated with Arthroscopic Palmer Classification. J Korean Soc Radiol 2011; 64: 265-271.

28. Wilson AJ, Gilula LA, Mann FA. Unidirectional joint communications in wrist arthrography: an evaluation of 250 cases. AJR Am J Roentgenol 1991; 157 (1): 105-109.

29. Wright TW, Del Charco M, Wheeler D. Incidence of ligament lesions and associated degenerative changes in the elderly wrist. J Hand Surg Am 1994; 19 (2): 313-318.

30. Shigematsu S, Abe M, Onomura T, Kinoshita M, Inoue T. Arthrography of the normal and posttraumatic wrist. J Hand Surg Am 1989; 14 (2 Pt 2): 410-412. DOI: 10.1016/0363-5023 (89)90126-3.

31. Linkous MD, Gilula LA. Wrist arthrography today. Radiol Clin North Am 1998; 36 (4): 651-672. DOI: 10.1016/s0033-8389 (05)70054-3.

Received March 16, 2021. Accepted April 22, 2021. 